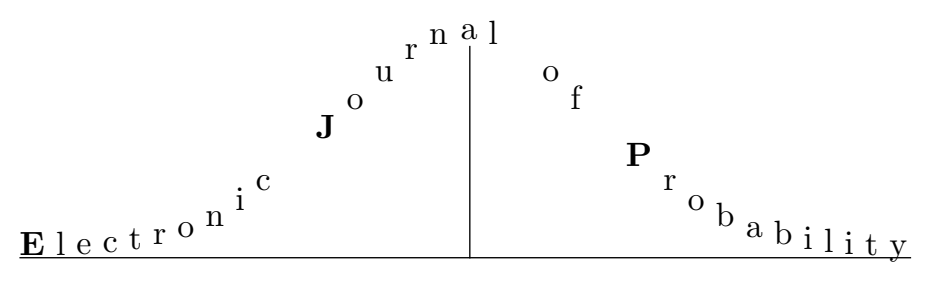

Vol. 11 (2006), Paper no. 13, pages 331-344.

Journal URL

http://www . math. washington.edu/ e jpecp/

\title{
Nonmonotonic coexistence regions for the two-type Richardson model on graphs
}

\author{
Maria Deijfen \\ Department of Mathematics, Stockholm University \\ 10691 Stockholm, Sweden \\ E-mail: mia@math.su.se. \\ Olle Häggström \\ Department of Mathematics \\ Chalmers University of Technology \\ 41296 Gothenburg, Sweden. \\ E-mail: olleh@math.chalmers.se.
}

\begin{abstract}
In the two-type Richardson model on a graph $\mathcal{G}=(\mathcal{V}, \mathcal{E})$, each vertex is at a given time in state 0,1 or 2 . A 0 flips to a 1 (resp. 2) at rate $\lambda_{1}\left(\lambda_{2}\right)$ times the number of neighboring 1 's (2's), while 1's and 2's never flip. When $\mathcal{G}$ is infinite, the main question is whether, starting from a single 1 and a single 2 , with positive probability we will see both types of infection reach infinitely many sites. This has previously been studied on the $d$-dimensional cubic lattice $\mathbb{Z}^{d}, d \geq 2$, where the conjecture (on which a good deal of progress has been made) is that such coexistence has positive probability if and only if $\lambda_{1}=\lambda_{2}$. In the present paper examples are given of other graphs where the set of points in the parameter space which admit such coexistence has a more surprising form. In particular, there exist graphs exhibiting coexistence at some value of $\frac{\lambda_{1}}{\lambda_{2}} \neq 1$ and non-coexistence when this ratio is brought closer to 1 .
\end{abstract}

Key words: Competing growth, graphs, coexistence.

AMS 2000 Subject Classification: Primary 60K35; Secondary: 82B43.

Submitted to EJP on March 1, 2005, final version accepted February 22, 2006. 


\section{Introduction}

The two-type Richardson model on a graph $\mathcal{G}=(\mathcal{V}, \mathcal{E})$ is an interacting particle system where at any time $t$, each vertex $v \in \mathcal{V}$ is in state 0,1 or 2 . Here 1 and 2 may be interpreted as two mutually exclusive types of infection, while sites in state 0 are thought of as not being infected. The dynamics is that a site $v \in \mathcal{V}$ in state 1 or 2 remains in this state forever, while a 0 flips to a 1 (resp. 2) at rate $\lambda_{1}\left(\lambda_{2}\right)$ times the number of 1's (2's) among the neighbors of $v$, where two sites are said to be neighbors if they share an edge in $\mathcal{E}$. Here $\lambda_{1}, \lambda_{2}>0$ are the two infection parameters of the model. The graph $\mathcal{G}$ will always be assumed to be countable and connected.

This model has previously been studied on the $\mathbb{Z}^{d}$ lattice, that is, on the graph whose vertex set is $\mathbb{Z}^{d}$ and whose edge set consists of all pairs of sites at Euclidean distance 1 from each other. The main question is whether, when we start from a single site in state 1 , a single site in state 2, and all others uninfected, we get positive probability for the event that both types of infection succeed in reaching an infinite number of sites. This event will in the following be referred to as infinite coexistence. Note that, given the initial configuration, the probability of infinite coexistence depends on $\lambda_{1}$ and $\lambda_{2}$ only through their ratio $\lambda=\frac{\lambda_{2}}{\lambda_{1}}$, as follows by a simple time-scaling argument. For this reason, we may without loss of generality set $\lambda_{1}=1$ and vary only $\lambda\left(=\lambda_{2}\right)$. The following conjecture goes back to Häggström and Pemantle [4, 5].

Conjecture 1.1 Infinite coexistence in the two-type Richardson model on $\mathbb{Z}^{d}, d \geq 2$, starting from a single infected site of each type, has positive probability if and only if $\lambda=1$.

A good deal of progress has been made on this conjecture. Häggström and Pemantle [4] showed that for $d=2$ and $\lambda=1$, infinite coexistence has positive probability. This result was recently extended to $d \geq 3$ (as well as to more general models) by Garet and Marchand [3] and independently by Hoffman [6]. As far as excluding infinite coexistence for $\lambda \neq 1$, the best result to date is the following.

Theorem 1.1 For the two-type Richardson model on $\mathbb{Z}^{d}, d \geq 2$, infinite coexistence has probability 0 for all but at most countably many values of $\lambda$.

An analogous result for a related continuum model was obtained by Deijfen et al. [2]. Also, Deijfen and Häggström [1] showed that the initial configuration, as long as there is a finite nonzero number of infected sites of each type and one infection has not already "strangled" the other, does not matter for the issue of whether or not infinite coexistence has positive probability. It is our experience from talking to colleagues about Theorem 1.1 (and its continuum analogue) that they tend to react with surprise at how weak this result is, and suggest that it should be easy to improve in such a way as to obtain the "only if" direction of Conjecture 1.1. Their argument is invariably the following.

Suppose for contradiction that infinite coexistence has positive probability for some $\lambda>1$. Then we can, due to Theorem 1.1, find some $\lambda^{\prime} \in(1, \lambda)$ for which infinite coexistence has probability 0 . But this is absurd, since

surely it must be easier to get infinite coexistence if we pick $\lambda$ closer to the symmetry point 1 . 
Although we do agree with this intuition (see also Lundin [7] for some numerical evidence in support for it), we think on the other hand that the claimed monotonicity may not be easy to prove. In particular, we do not believe that it is possible to establish using abstract arguments that disregard the particular geometry of the $\mathbb{Z}^{d}$ lattice.

It is the purpose of this paper to support this point of view by giving examples of other graphs, where the two-type Richardson model behaves in a way that conflicts with the intuition about monotonicity in $\lambda$. These graphs differ from the $\mathbb{Z}^{d}$-lattice in that they are highly non-symmetric: certain parts of the graph are designed specifically with propagation of type- 1 infection in mind, while other (different) parts are meant for type-2 infection.

For a graph $\mathcal{G}$, write $\operatorname{Coex}(\mathcal{G})$ for the set of all $\lambda \geq 1$ such that there exists an initial configuration $\xi \in\{0,1,2\}^{\mathcal{V}}$ which has only finitely many infected sites of each type and for which the two-type Richardson model starting from $\xi$ yields infinite coexistence with positive probability. (Note that by time-scaling and interchange of 1's and 2's, coexistence is possible for $\lambda$ if and only if it is possible for $\lambda^{-1}$; hence no information is lost by restricting to $\lambda \geq 1$.) In Sections 2 and 3 , we will exhibit examples of graphs $\mathcal{G}$ that demonstrate that, among others, the following kinds of coexistence sets $\operatorname{Coex}(\mathcal{G})$ are possible:

- For any positive integer $k, \operatorname{Coex}(\mathcal{G})$ may consist of exactly $k$ points.

- $\operatorname{Coex}(\mathcal{G})$ may be countably infinite.

- $\operatorname{Coex}(\mathcal{G})$ may be an interval $(a, b)$ with $1<a<b$.

Note that all three examples show that the monotonicity intuition suggested in (1) fails for general graphs. However, as mentioned above, all examples will be highly non-symmetric. A reasonable guess is that the intuition in (1) is in fact correct on transitive graphs.

As a complement to these examples, we will end the paper by giving a positive result (Theorem 4.1) ruling out a large class of more exotic coexistence regions, such as those that are uncountable with zero Lebesgue measure.

Before moving on to the examples and results, let us say a few words about the construction and the well-definedness of the two-type Richardson model on graphs. To this end, let $S_{t}^{1}$ and $S_{t}^{2}$ denote the set of type 1 and 2 infected vertices respectively at time $t$, and, for a vertex set $A \in \mathcal{V}$, write $\partial A$ for the set of edges with one endpoint in $A$ and one endpoint in $A^{c}$. One way to construct the model with parameters $\lambda_{1}, \lambda_{2}>0$ is to assign i.i.d. exponential random variables $\{X(e)\}_{e \in \mathcal{E}}$ with mean 1 to the edges of $\mathcal{G}$ and update the sets $S_{t}^{1}$ and $S_{t}^{2}$ inductively at discrete time points $\left\{T_{n}\right\}$ as follows:

1. Define $T_{0}=0$ and pick two bounded initial sets $S_{0}^{1}$ and $S_{0}^{2}$.

2. For $n \geq 1$, given $T_{n-1}, S_{T_{n-1}}^{1}$ and $S_{T_{n-1}}^{2}$, define $T_{n}=\min \left\{T_{n}^{1}, T_{n}^{2}\right\}$, where

$$
T_{n}^{i}=\inf \left\{\lambda_{i} X(e) ; e \in \partial S_{T_{n-1}}^{i} \backslash \partial S_{T_{n-1}}^{j}\right\}
$$

with $i, j \in\{1,2\}$ and $i \neq j$. 
3. Let $S_{t}^{i}=S_{T_{n-1}}^{i}$ for $t \in\left[T_{n-1}, T_{n}\right)$ and $i=1,2$. Then, at time $T_{n}$, the sets are updated in that infection is transferred through the edge defining $T_{n}$. More precisely, if $T_{n}=T_{n}^{1}$ and $x$ is the uninfected end of the edge where the infimum in the definition of $T_{n}^{1}$ is attained, then $S_{T_{n}}^{1}=S_{T_{n-1}}^{1} \cup\{x\}$ and $S_{T_{n}}^{2}=S_{T_{n-1}}^{2}$. Similarly, if $T_{n}=T_{n}^{2}$ then $S_{T_{n-1}}^{2}$ is updated analogously, while $S_{T_{n-1}}^{1}$ is left unchanged.

If $\mathcal{G}$ has bounded degree (as all our examples will) and if initially only finitely many vertices are infected, then it is straightforward to see that almost surely no explosion will occur (where explosion means that infinitely many transitions take place in finite time), and that the process is Markovian with the desired infection intensities.

\section{Basic examples}

We begin with our simplest example: a graph $\mathcal{G}$ that admits infinite coexistence in the two-type Richardson model if and only if $\lambda$ is $\frac{1}{2}$ or 2 .

Proposition 2.1 There exists a graph $\mathcal{G}$ with $\operatorname{Coex}(\mathcal{G})=\{2\}$.

Proof. The graph we use to prove this result will look a bit like a ladder: with two "spines" linked by a number of bridges.

More specifically, let $\left\{v_{1, j}\right\}_{j \geq 0}$ and $\left\{v_{2, j}\right\}_{j \geq 0}$ be two sequences of vertices, each internally linked by edges $\left\{e_{1, j}\right\}_{j \geq 0}:=\left\{\left\langle v_{1, j}, v_{1, j+1}\right\rangle\right\}_{j \geq 0}$ and $\left\{e_{2, j}\right\}_{j \geq 0}:=\left\{\left\langle v_{2, j}, v_{2, j+1}\right\rangle\right\}_{j \geq 0}$, respectively. These two infinite paths will be linked to each other by finite paths, called bridges, where the $n$ :th such bridge emanates from $v_{1, a_{n}}$ and arrives at $v_{2,2 a_{n}}$. Here $\left(a_{1}, a_{2}, \ldots\right)$ is a rapidly increasing sequence of positive integers (how rapidly will be indicated later). The $n$ :th bridge will be called $B_{n}$ and have length $\left\lceil a_{n}^{7 / 8}\right\rceil$, where $\lceil\cdot\rceil$ denotes rounding up to the nearest integer.

Now consider the two-type Richardson model on this graph with infection rates $\lambda_{1}=1$ and $\lambda_{2}=\lambda>0$ starting with a single infected 1 at $v_{1,0}$, and a single infected 2 at $v_{2,0}$. It is easy to see that if a site on the first spine $\left\{v_{1, j}\right\}_{j \geq 0}$ is ever infected by the type 2 infection, then type 1 is strangled (that is, it is cut off from the possibility of ever infecting more than a finite number of sites). Hence, the event $C_{1}$ of infinite growth of the type 1 infection happens if and only if all sites on the first spine are eventually infected by type 1 . Similarly, the event $C_{2}$ of infinite growth of the type 2 infection happens if and only if all sites on the second spine are eventually infected by type 2 .

With the edge representation indicated at the end of Section 1, let $D_{1, n}$ denote the event that

$$
\sum_{j=0}^{a_{n}-1} X\left(e_{1, j}\right)>\sum_{j=0}^{2 a_{n}-1} \lambda^{-1} X\left(e_{2, j}\right)+\sum_{e \in B_{n}} \lambda^{-1} X(e) .
$$

Note that, unless the type 1 infection has already managed to infect some site on the second spine before reaching $v_{1, a_{n}}$, the site $v_{1, a_{n}}$ gets type 1 infected if and only if $D_{1, m}$ does not happen for any $m \leq n$. Analogously, define $D_{2, n}$ as

$$
\sum_{j=0}^{2 a_{n}-1} \lambda^{-1} X\left(e_{2, j}\right)>\sum_{j=0}^{a_{n}-1} X\left(e_{1, j}\right)+\sum_{e \in B_{n}} X(e),
$$


and note that, if type 2 has not already infected some site on the first spine before reaching $v_{2,2 a_{n}}$, then $v_{2,2 a_{n}}$ gets type 2 infected if and only if $D_{2, n}$ does not happen. Hence, the event $C=C_{1} \cap C_{2}$ of infinite coexistence happens if and only if none of the events $D_{1,1}, D_{1,2}, \ldots$ and $D_{2,1}, D_{2,2}, \ldots$ happen.

To get a grip on the probabilities of these events, it is useful to introduce the variables

$$
T_{1, n}=\sum_{j=0}^{2 a_{n}-1} \lambda^{-1} X\left(e_{2, j}\right)+\sum_{e \in B_{n}} \lambda^{-1} X(e)-\sum_{j=0}^{a_{n}-1} X\left(e_{1, j}\right)
$$

and

$$
T_{2, n}=\sum_{j=0}^{a_{n}-1} X\left(e_{1, j}\right)+\sum_{e \in B_{n}} X(e)-\sum_{j=0}^{2 a_{n}-1} \lambda^{-1} X\left(e_{2, j}\right)
$$

and note that $D_{1, n}=\left\{T_{1, n}<0\right\}$ and $D_{2, n}=\left\{T_{2, n}<0\right\}$. The expectation and variance of $T_{1, n}$ are

$$
\mathbf{E}\left[T_{1, n}\right]=\left(2 \lambda^{-1}-1\right) a_{n}+\lambda^{-1}\left\lceil a_{n}^{7 / 8}\right\rceil
$$

and

$$
\operatorname{Var}\left[T_{1, n}\right]=\left(2 \lambda^{-2}+1\right) a_{n}+\lambda^{-2}\left\lceil a_{n}^{7 / 8}\right\rceil
$$

and for $T_{2, n}$ we get

$$
\mathbf{E}\left[T_{2, n}\right]=\left(1-2 \lambda^{-1}\right) a_{n}+\left\lceil a_{n}^{7 / 8}\right\rceil
$$

and

$$
\operatorname{Var}\left[T_{2, n}\right]=\left(1+2 \lambda^{-2}\right) a_{n}+\left\lceil a_{n}^{7 / 8}\right\rceil .
$$

There are now three cases to consider separately, namely $\lambda>2, \lambda=2$ and $\lambda<2$.

We begin with the case $\lambda>2$. We then have $2 \lambda^{-1}-1<0$, meaning that $\mathbf{E}\left[T_{1, n}\right]<0$ for $a_{n}$ large enough. Writing $\neg$ for set complement, Chebyshev's inequality gives

$$
\begin{aligned}
\mathbf{P}\left(\neg D_{1, n}\right) & =\mathbf{P}\left(T_{1, n} \geq 0\right) \\
& \leq \frac{\operatorname{Var}\left[T_{1, n}\right]}{\left(\mathbf{E}\left[T_{1, n}\right]\right)^{2}} \\
& =\frac{\left(2 \lambda^{-2}+1\right) a_{n}+\lambda^{-2}\left\lceil a_{n}^{7 / 8}\right\rceil}{\left(\left(2 \lambda^{-1}-1\right) a_{n}+\lambda^{-1}\left\lceil a_{n}^{7 / 8}\right\rceil\right)^{2}}
\end{aligned}
$$

which tends to 0 as $a_{n} \rightarrow \infty$, and, since $a_{n}$ tends to $\infty$ with $n$, it follows that $\lim _{n \rightarrow \infty} \mathbf{P}\left[D_{1, n}\right]=$ 1. Hence

$$
\begin{aligned}
\mathbf{P}(C) & \leq \mathbf{P}\left(C_{1}\right) \\
& =1-\mathbf{P}\left(\bigcup_{n=1}^{\infty} D_{1, n}\right) \\
& \leq 1-\lim _{n \rightarrow \infty} \mathbf{P}\left(D_{1, n}\right) \\
& =0 .
\end{aligned}
$$


In the case $\lambda<2$, we have $1-2 \lambda^{-1}<0$ and hence $\mathbf{E}\left[T_{2, n}\right]<0$ for $a_{n}$ large enough, whence analogously to (4) we get

$$
\mathbf{P}\left(\neg D_{2, n}\right) \leq \frac{\left(1+2 \lambda^{-2}\right) a_{n}+\left\lceil a_{n}^{7 / 8}\right\rceil}{\left(\left(1-2 \lambda^{-1}\right) a_{n}+\left\lceil a_{n}^{7 / 8}\right\rceil\right)^{2}}
$$

This tends to 0 as $a_{n} \rightarrow \infty$, so that $\lim _{n \rightarrow \infty} \mathbf{P}\left(D_{2, n}\right)=1$, implying that

$$
\begin{aligned}
\mathbf{P}(C) & \leq \mathbf{P}\left(C_{2}\right) \\
& =1-\mathbf{P}\left(\bigcup_{n=1}^{\infty} D_{2, n}\right) \\
& \leq 1-\lim _{n \rightarrow \infty} \mathbf{P}\left(D_{2, n}\right) \\
& =0 .
\end{aligned}
$$

The final case $\lambda=2$ is slightly more subtle. Both $\mathbf{E}\left[T_{1, n}\right]$ and $\mathbf{E}\left[T_{2, n}\right]$ are then positive for any $n$, and another application of Chebyshev's inequality gives

$$
\begin{aligned}
\mathbf{P}\left(D_{1, n}\right) & =\mathbf{P}\left(T_{1, n}<0\right) \\
& \leq \frac{\operatorname{Var}\left[T_{1, n}\right]}{\left(\mathbf{E}\left[T_{1, n}\right]\right)^{2}} \\
& =\frac{\left(2^{-1}+1\right) a_{n}+2^{-2}\left\lceil a_{n}^{7 / 8}\right\rceil}{\left(2^{-1}\left\lceil a_{n}^{7 / 8}\right\rceil\right)^{2}}
\end{aligned}
$$

which tends to 0 as $a_{n} \rightarrow \infty$. Similarly, $\mathbf{P}\left(D_{2, n}\right) \rightarrow 0$ as $a_{n} \rightarrow \infty$. So far we have not specified how quickly the numbers $a_{n}$ tends to $\infty$ with $n$. We are therefore free to choose $\left(a_{1}, a_{2}, \ldots\right)$ in such a way that

$$
\sum_{n=1}^{\infty}\left(\mathbf{P}\left(D_{1, n}\right)+\mathbf{P}\left(D_{2, n}\right)\right)<1 .
$$

With such a choice of $\left(a_{1}, a_{2}, \ldots\right)$, we get

$$
\begin{aligned}
\mathbf{P}(C) & =1-\mathbf{P}\left(\neg C_{1} \cup \neg C_{2}\right) \\
& =1-\mathbf{P}\left(\bigcup_{n=1}^{\infty} D_{1, n} \cup \bigcup_{n=1}^{\infty} D_{2, n}\right) \\
& \geq 1-\sum_{n=1}^{\infty}\left(\mathbf{P}\left(D_{1, n}\right)+\mathbf{P}\left(D_{2, n}\right)\right) \\
& >0 .
\end{aligned}
$$

Having worked through the three cases $\lambda>2, \lambda=2$ and $\lambda<2$, we have now shown that with the given initial condition (infection 1 at $v_{1,0}$ and infection 2 at $v_{2,0}$ ), infinite coexistence has positive probability if and only if $\lambda=2$. In order to prove Proposition 2.1, it remains to show that no other finite initial condition can yield infinite coexistence for any other $\lambda \geq 1$. 
Of course, if infections 1 and 2 switch places in the above initial condition, then infinite coexistence has positive probability if and only if $\lambda=\frac{1}{2}$. For other initial conditions, note that infinite coexistence implies that either

(i) infection 1 finds a path to $\infty$ which from some point onwards belongs to the first spine, and infection 2 similarly reaches $\infty$ along the second spine, or

(ii) vice versa.

But the above analysis shows that scenario (i) requires $\lambda=2$, and similarly scenario (ii) requires $\lambda=\frac{1}{2}$, so $\operatorname{Coex}(\mathcal{G})=\{2\}$ as desired.

The reason we get a different behavior for the competition process here as compared to on $\mathbb{Z}^{d}$ is related to the lack of symmetry of the above graph. The two infinite spines provide two separate paths to infinity on which the infection types can keep in step with each other if their intensities are chosen to match the density of vertices on the spines. Note also that, on the above graph, the initial configuration is indeed important for the possibility of infinite coexistence. For instance, as pointed out by the end of the proof of Proposition 2.1, switching two single sources might change the coexistence probability. Again, this is related to the lack of symmetry of the graph and contrasts with the $\mathbb{Z}^{d}$ case (on $\mathbb{Z}^{d}$, switching two sources located at $x$ and $y$ respectively does not affect the coexistence probability, since there exists an automorphism of the graph $\mathbb{Z}^{d}$ that exchanges $x$ and $y$ ).

Of course, for any $\alpha \geq 1$, we can modify the above construction by letting bridges connect $v_{1, a_{n}}$ and $v_{2,\left[\alpha a_{n}\right\rceil}$ rather than $v_{1, a_{n}}$ and $v_{2,2 a_{n}}$, thereby obtaining a graph $\mathcal{G}$ with $\operatorname{Coex}(\mathcal{G})=\{\alpha\}$. See Theorem 3.1 for a more general result.

Next, we show how to turn $\operatorname{Coex}(\mathcal{G})$ into an entire interval.

Proposition 2.2 There exists a graph $\mathcal{G}$ for which $\operatorname{Coex}(\mathcal{G})$ equals the interval $[2,5]$.

Proof. As in the proof of Proposition 2.1, we take $\mathcal{G}$ to consist of two spines $\left\{v_{1, j}\right\}_{j \geq 0}$ and $\left\{v_{2, j}\right\}_{j \geq 0}$, together with a sequence of bridges between them. This time, we take the $n$ 'th bridge $B_{n}$ to begin at $v_{1, a_{n}}$ and end at $v_{2,4 a_{n}}$, and to have length $a_{n}+\left[a_{n}^{7 / 8}\right]$.

Note that (i) and (ii) in the proof of Proposition 2.1 are the only two possible scenarios for infinite coexistence, and that it is therefore sufficient to consider the initial condition with a single 1 at $v_{1,0}$ and a single 2 at $v_{2,0}$. With this initial condition, the same kind of applications of Chebyshev's inequality as in the proof of Proposition 2.1 show that $\mathbf{P}\left(\cap_{n=1}^{\infty} D_{1, n}^{c}\right)=0$ for $\lambda>5$, that $\mathbf{P}\left(\cap_{n=1}^{\infty} D_{2, n}^{c}\right)=0$ for $\lambda<2$, and that $\mathbf{P}(C)>0$ for $\lambda \in[2,5]$ provided the sequence $\left(a_{1}, a_{2}, \ldots\right)$ grows sufficiently fast. Thus, $\operatorname{Coex}(\mathcal{G})=[2,5]$.

Several variations of Proposition 2.2 are easily obtained, such as the following (and combinations thereof):

1. For any $1 \leq \alpha<\alpha^{\prime}$, the coexistence region $[2,5]$ can be replaced by $\operatorname{Coex}(\mathcal{G})=\left[\alpha, \alpha^{\prime}\right]$ by noting that, if the bridge $B_{n}$ is taken to begin at $v_{1, a_{n}}$ and end at $v_{2,\left\lceil\gamma a_{n}\right\rceil}$ and to have length $\left[\beta a_{n}+a_{n}^{7 / 8}\right]$, then infinite coexistence is possible for $\lambda \in[\gamma /(1+\beta), \gamma+\beta]$. The interval $\left[\alpha, \alpha^{\prime}\right]$ is hence obtained by letting $B_{n}$ end at

$$
v_{2,\left\lceil\frac{\alpha\left(1+\alpha^{\prime}\right) a_{n}}{1+\alpha}\right\rceil},
$$


and have length $\left\lceil\frac{\left(\alpha^{\prime}-\alpha\right) a_{n}}{1+\alpha}\right\rceil+\left\lceil a_{n}^{7 / 8}\right\rceil$.

2. If the $B_{n}$ 's are taken to have length $a_{n}-\left\lceil a_{n}^{7 / 8}\right\rceil$ rather than $a_{n}+\left\lceil a_{n}^{7 / 8}\right\rceil$, then the coexistence region $[2,5]$ is replaced by the open interval $(2,5)$.

3. If the lengths of the $B_{n}$ 's are taken to be $a_{n}$ (with no lower order correction), start from $v_{1, a_{n}}$ and end at

$$
v_{2,3 a_{n}+\left\lceil a_{n}^{7 / 8}\right\rceil,}
$$

then we get $\operatorname{Coex}(\mathcal{G})=(2,5]$. The other half-open interval $[2,5)$ can be obtained analogously.

\section{$3 \quad$ Further examples}

The following result generalizes Proposition 2.1.

Theorem 3.1 For any $k$ and any $\alpha_{1}, \ldots, \alpha_{k} \in[1, \infty)$, there exists a graph $\mathcal{G}$ with $\operatorname{Coex}(\mathcal{G})=$ $\left\{\alpha_{1}, \ldots, \alpha_{k}\right\}$.

Intuitively, it is not hard to figure out what kind of modification of the example in Proposition 2.1 that would lead to Theorem 3.1. Let $\mathcal{G}$ contain $k+1$ spines, whose respective vertex sets we may denote $\left\{v_{\text {main, } j}\right\}_{j \geq 0}$ and $\left\{v_{1, j}\right\}_{j \geq 0}, \ldots,\left\{v_{k, j}\right\}_{j \geq 0}$. As before, $\left(a_{1}, a_{2}, \ldots\right)$ will be a rapidly increasing sequence, and for each $n$ there will be bridges $B_{1, n}, \ldots, B_{k, n}$, each with length $\left\lceil a_{n}^{7 / 8}\right\rceil$, bridge $B_{m, n}$ starting at $v_{\text {main, } a_{n}}$ and ending at $v_{m,\left[\alpha_{m} a_{n}\right\rceil}$.

Infinite coexistence should now be possible for $\lambda=\alpha_{m}$ by means of infection 1 taking over the main spine $\left\{v_{\text {main, } j}\right\}_{j \geq 0}$, and infection 2 taking over the $m$ 'th auxiliary spine $\left\{v_{m, j}\right\}_{j \geq 0}$ (while all other spines are conquered by infection 1$)$. On the other hand, no such scenario seems possible when $\lambda \geq 1$ is not an element of $\left\{\alpha_{1}, \ldots, \alpha_{k}\right\}$.

Proving this turns out to be a technically somewhat more challenging task compared to what we did in Section 2, the reason being that there is no slick decription of the possible ways of infinite coexistence like (i) and (ii) in the proof of Proposition 2.1. On the contrary, for $k \geq 2$ we may (in principle) imagine an infinite coexistence scenario where each infection "zig-zags" between the main spine and the other spines in a more or less complicated manner. For this reason, we choose to construct the graph in a more iterative fashion, and to base our arguments on the following lemma. For the two-type Richardson model on a graph $\mathcal{G}=(\mathcal{V}, \mathcal{E})$ and a vertex $v \in \mathcal{V}$, write $T_{v}$ for the time at which $v$ becomes infected.

Lemma 3.1 Consider the two-type Richardson model with infection rates $\lambda_{1}=1$ and $\lambda_{2}=$ $\lambda \geq 1$ on a graph $\mathcal{G}$ constructed as follows: Let $\mathcal{G}^{\prime}=\left(\mathcal{V}^{\prime}, \mathcal{E}^{\prime}\right)$ be an arbitrary finite graph with $k \geq 1$ distinguished vertices $v_{1}, \ldots, v_{k} \in \mathcal{V}^{\prime}$, and obtain $\mathcal{G}$ by, for $i=1, \ldots, k$, attaching to $v_{i}$ an infinite path with vertex set $\left\{v_{i, j}\right\}_{j \geq 1}$.

Fix $\varepsilon>0$. Then, for all sufficiently large $N$ (depending on $\varepsilon$ but not on $\lambda$ ), we have that for any $\xi \in\{1,2\}^{\left\{v_{1}, \ldots, v_{k}\right\}}$ and any initial condition such that

(a) only vertices in $\mathcal{G}^{\prime}$ are initially infected, and 
(b) the event $A_{\xi}$ that the infection proceeds in such a way that for each $i \in\{1, \ldots, k\}$, vertex $v_{i}$ eventually gets infection $\xi\left(v_{i}\right)$, has positive probability,

the following holds for each $i=\{1, \ldots, k\}$ :

$$
\mathbf{P}\left(T_{v_{i, N}} \in\left(\lambda^{1-\xi\left(v_{i}\right)} N-N^{3 / 4}, \lambda^{1-\xi\left(v_{i}\right)} N+N^{3 / 4}\right) \mid A_{\xi}\right) \geq 1-\varepsilon
$$

Proof. If the initial condition is such that $v_{1}, \ldots, v_{k}$ are all initially infected, then the lemma is immediate from the Central Limit Theorem applied to the sum

$$
X\left(\left\langle v_{i}, v_{i, 1}\right\rangle\right)+\sum_{j=1}^{N-1} X\left(\left\langle v_{i, j}, v_{i, j+1}\right\rangle\right)
$$

for each $i \in\{1, \ldots, k\}$. For other initial conditions, note that, since $\mathcal{G}^{\prime}$ is finite and connected, the time $T_{v_{i}}$ when the vertex $v_{i}$ is infected is almost surely finite, implying that

$$
P\left(T_{v_{i}} \leq N^{1 / 8} \mid A_{\xi}\right) \geq 1-\varepsilon / 2
$$

for large $N$. On $A_{\xi}$, the vertex $v_{i}$ is infected by the type $\xi\left(v_{i}\right)$ infection and the Central Limit Theorem applied to the same sum as above hence gives that

$$
\mathbf{P}\left(T_{v_{i, N}}-T_{v_{i}} \in\left(\lambda^{1-\xi\left(v_{i}\right)} N-N^{3 / 4}, \lambda^{1-\xi\left(v_{i}\right)} N+N^{5 / 8}\right) \mid A_{\xi}\right) \geq 1-\varepsilon / 2 .
$$

The desired estimate now follows by taking $N$ large enough to ensure that both (5) and (6) hold for all $i \in\{1, \ldots, k\}$.

Proof of Theorem 3.1. Fix the coexistence set $\left\{\alpha_{1}, \ldots \alpha_{k}\right\}$ that we are trying to obtain, and define $\alpha_{0}=1$ and $\alpha_{\max }=\max \left\{\alpha_{1}, \ldots, \alpha_{k}\right\}$. The graph $\mathcal{G}=(\mathcal{V}, \mathcal{E})$ that will serve as an example, will be obtained from a sequence of graphs $\left\{\mathcal{G}_{n}=\left(\mathcal{V}_{n}, \mathcal{E}_{n}\right)\right\}_{n \geq 0}$ which is increasing in the sense that

$$
\mathcal{V}_{0} \subseteq \mathcal{V}_{1} \subseteq \cdots
$$

and

$$
\mathcal{E}_{0} \subseteq \mathcal{E}_{1} \subseteq \cdots
$$

The "limiting" graph $\mathcal{G}=(\mathcal{V}, \mathcal{E})$ is then given by $\mathcal{V}=\bigcup_{n=0}^{\infty} \mathcal{V}_{n}$ and $\mathcal{E}=\bigcup_{n=0}^{\infty} \mathcal{E}_{n}$. The graphs $\mathcal{G}_{n}$ are defined inductively as follows.

Let $\left(b_{1}, b_{2}, \ldots\right)$ be a rapidly growing sequence of positive integers; how rapidly $b_{n} \rightarrow \infty$ with $n$ will be specified later. The construction begins with taking $\mathcal{G}_{0}$ to be the complete graph on $k+1$ vertices $x_{0,0}, x_{0,1}, \ldots, x_{0, k}$. For the induction step, suppose that we have the graph $\mathcal{G}_{n}$ with $k+1$ distinguished vertices $x_{n, 0},, x_{n, 1}, \ldots, x_{n, k}$, and let $\mathcal{G}_{n+1}$ be the graph obtained from $\mathcal{G}_{n}$ by the following amendments:

(a) for $i=0, \ldots, k$, attach to $x_{n, i}$ a path of length $\left\lceil\alpha_{i} b_{n+1}\right\rceil$, denoting the last vertex of this path by $x_{n+1, i}$, and 
(b) for $i=1, \ldots, k$, link $x_{n+1,0}$ and $x_{n+1, i}$ by a path (called a bridge and denoted $B_{n+1, i}$ ) of length $\left\lceil b_{n+1}^{7 / 8}\right\rceil$.

This defines $\left(\mathcal{G}_{0}, \mathcal{G}_{1}, \ldots\right)$ and $\mathcal{G}$, apart from that the sequence $\left(b_{1}, b_{2}, \ldots\right)$ has not been specified. In order to make that choice, begin by specifying two decreasing sequences $\left(\delta_{1}, \delta_{2}, \ldots\right)$ and $\left(\varepsilon_{1}, \varepsilon_{2}, \ldots\right)$ of positive numbers tending to 0 , with the second sequence having the additional property that

$$
\sum_{n=1}^{\infty} \varepsilon_{n}<\frac{1}{k+2}
$$

Given $b_{1}, \ldots, b_{n}$ (and, thus, $\mathcal{G}_{1}, \ldots, \mathcal{G}_{n}$ ), pick $b_{n+1}$ large enough so that the following conditions hold:

(i) For $\xi \in\{1,2\}^{\left\{x_{n, 0}, \ldots, x_{n, k}\right\}}$, let $A_{n, \xi}$ denote the event that $x_{n, 0}, \ldots, x_{n, k}$ are infected by type $\xi\left(x_{n, 0}\right), \ldots, \xi\left(x_{n, k}\right)$, respectively. For an arbitrary such $\xi$ and arbitrary initial conditions that are confined to $\mathcal{G}_{n}$ and that makes it possible for $A_{n, \xi}$ to happen before any vertices outside $\mathcal{G}_{n}$ are infected, we have for $i=0, \ldots, k$ and any $\lambda \geq 1$ that

$$
\begin{gathered}
\mathbf{P}\left(T_{x_{n+1}, i} \in\left(\lambda^{1-\xi\left(x_{n, i}\right)} \alpha_{i} b_{n+1}-\alpha_{i} b_{n+1}^{3 / 4}, \lambda^{1-\xi\left(x_{n, i}\right)} \alpha_{i} b_{n+1}+\alpha_{i} b_{n+1}^{3 / 4}\right) \mid A_{n, \xi}\right) \\
\geq 1-\varepsilon_{n+1}
\end{gathered}
$$

(note that this holds for $b_{n+1}$ large enough, due to Lemma 3.1).

(ii)

(iii)

$$
2 \alpha_{\max } b_{n+1}^{3 / 4}<\frac{\delta_{n+1} b_{n+1}}{2}
$$

$$
\mathbf{P}\left(\sum_{e \in B_{n+1, i}} X(e) \in\left(2 \alpha_{\max } b_{n+1}^{3 / 4}, \frac{\delta_{n+1} b_{n+1}}{2}\right) \text { for } i=1, \ldots, k\right) \geq 1-\varepsilon_{n+1}
$$

(note that this holds for $b_{n+1}$ large enough, since by the Weak Law of Large Numbers the $\operatorname{sum} \sum_{e \in B_{n+1, i}} X(e)$ is concentrated around $b_{n+1}^{7 / 8}$ for large values of $\left.b_{n+1}\right)$.

This specifies $\mathcal{G}$. Now fix $i \in\{1, \ldots, k\}$, and consider the two-type Richardson model with $\lambda_{1}=1$ and $\lambda=\lambda_{2}=\alpha_{i}$, starting with vertex $x_{0, i}$ in state 2 , vertices $x_{0,0}, x_{0,1}, \ldots, x_{0, i-1}, x_{0, i+1}, \ldots, x_{0, k}$ in state 1 , and all other vertices uninfected.

For $n=1,2, \ldots$, let $D_{n, i}$ denote the event that $x_{n, 0}$ eventually gets type 1 infected, that $x_{n, i}$ eventually gets type 2 infected and that, at the time when the last one (in time) of $x_{n, 0}$ and $x_{n, i}$ is infected, there is no type 2 infection on any of the spines $j \neq i$. We have that

$$
\mathbf{P}\left(D_{1, i}\right) \geq 1-(k+2) \varepsilon_{1}
$$

because the choice of $b_{1}$ implies that with probability at least $1-(k+2) \varepsilon_{1}$, the type 1 infection reaches $x_{n, 0}$ before the type 2 infection has crossed the bridge $B_{1, i}$ and the type 2 infection reaches $x_{n, i}$ before the type 1 infection has crossed $B_{1, i}$. 
More generally, for any $n$, it follows from the choice of $b_{n+1}$ that

$$
\mathbf{P}\left(D_{n+1, i} \mid D_{n, i}\right) \geq 1-(k+2) \varepsilon_{n+1} .
$$

Hence, using (7), we get

$$
\begin{aligned}
\mathbf{P}(C) & \geq \mathbf{P}\left(\liminf _{n \rightarrow \infty} D_{n, i}\right) \\
& \geq \liminf _{n \rightarrow \infty} \mathbf{P}\left(D_{n, i}\right) \\
& \geq \prod_{n=1}^{\infty}\left(1-(k+2) \varepsilon_{n}\right) \\
& \geq 1-\sum_{n=1}^{\infty}(k+2) \varepsilon_{n} \\
& >0 .
\end{aligned}
$$

We have thus shown that $\alpha_{i} \in \operatorname{Coex}(\mathcal{G})$ for each $i \in\{1, \ldots, k\}$.

What remains is to show that for any $\lambda \geq 1$ such that $\lambda \notin\left\{\alpha_{1}, \ldots, \alpha_{k}\right\}$, and any finite initial condition, we have that $\mathbf{P}(C)=0$. We will assume for the moment that $\lambda>1$; fix such a $\lambda \notin\left\{\alpha_{1}, \ldots, \alpha_{k}\right\}$ and an arbitrary initial condition, and pick $n$ large enough so that

(a) the set of infected sites in the initial condition is confined to $\mathcal{G}_{n}$

(b) $\left|\lambda-\alpha_{i}\right|>\delta_{n+1}$ for all $i \in\{0, \ldots, k\}$, and

(c) $\frac{\left|\lambda-\alpha_{i}\right|-\delta_{n+1}}{\lambda} b_{n+1}>\left(\alpha_{i}+1\right) b_{n+1}^{3 / 4}$ for all $i \in\{0, \ldots, k\}$.

We now claim that

$$
\mathbf{P}\left(\text { both types of infection reach }\left\{x_{n+1,0}, \ldots, x_{n+1, k}\right\}\right) \leq(k+2) \varepsilon_{n+1} .
$$

Once this is established we are done, because $\mathbf{P}(C)$ is bounded by the left-hand side of (8) while the right-hand side can be made arbitrarily small by picking $n$ even larger.

To show that (8) holds, it suffices to show that it holds even if we condition on the types of infection $\xi=\left(\xi\left(x_{n, 0}\right), \ldots, \xi\left(x_{n, k}\right)\right)$ that reach $x_{n, 0}, \ldots, x_{n, k}$. Given such a $\xi$ (with both types of infection appearing), define

$$
\alpha_{\xi}=\min \left\{\alpha_{i}: i \in\{0,1, \ldots, k\}, \xi\left(x_{n, i}\right)=2\right\}
$$

and then fix $i$ in such a way that $\alpha_{\xi}=\alpha_{i}$. By the choice of $n$ we have that $\left|\lambda-\alpha_{\xi}\right|>\delta_{n+1}$. There are now two cases to consider: $\lambda>\alpha_{\xi}$ and $\lambda<\alpha_{\xi}$. In the former case $\lambda>\alpha_{\xi}$, we have by the choice of $b_{n+1}$ that with conditional probability at least $1-(k+2) \varepsilon_{n+1}$, infection 2 claims all of $x_{n+1,0}, \ldots, x_{n+1, k}$ (by rushing from $x_{n, i}$ to $x_{n+1, i}$, then across the bridge $B_{n+1, i}$, and then across the $k-1$ other bridges emanating from $x_{n+1,0}$, all of this before infection 1 arrives at any of the $x_{n+1, j}$ 's). Similarly, in the latter case $\lambda<\alpha_{\xi}$, we get by the choice of $b_{n+1}$ that with conditional probability at least $1-(k+2) \varepsilon_{n+1}$, infection 1 claims all of $x_{n+1,0}, \ldots, x_{n+1, k}$ (by rushing from $x_{n, 0}$ to $x_{n+1,0}$ and then across all bridges emanating from $\left.x_{n+1,0}\right)$. Thus, (8) is established when $\lambda>1$. 
It only remains to deal with the case $\lambda=1 \notin\left\{\alpha_{1}, \ldots, \alpha_{k}\right\}$. This follows by a similar argument: given $\xi=\left(\xi\left(x_{n, 0}\right), \ldots, \xi\left(x_{n, k}\right)\right)$, the infection that has captured $x_{n, 0}$ will, with probability at least $1-(k+2) \varepsilon_{n+1}$, capture all of $x_{n+1,0}, \ldots, x_{n+1, k}$.

It now only takes a minor extension of the above construction to get a graph that can be used to establish the following result.

Theorem 3.2 There exists a graph $\mathcal{G}$ for which $\operatorname{Coex}(\mathcal{G})$ is countably infinite.

Proof. Take $\left(\alpha_{1}, \alpha_{2}, \ldots\right)$ to be an unbounded and strictly increasing sequence with $\alpha_{1} \geq 1$. We will construct a graph with $\operatorname{Coex}(\mathcal{G})=\left\{\alpha_{1}, \alpha_{2}, \ldots\right\}$. As in the proof of Theorem 3.1, take $\left(b_{1}, b_{2}, \ldots\right)$ to be a rapidly growing sequence of positive integers, and construct $\mathcal{G}=(\mathcal{V}, \mathcal{E})$ as a limit of an increasing sequence $\left\{\mathcal{G}_{n}=\left(\mathcal{E}_{n}, \mathcal{V}_{n}\right)\right\}_{n \geq 0}$ of finite graphs.

Take $\mathcal{G}_{0}$ to consist of a single vertex $x_{0,0}$, and proceed inductively: Given $\mathcal{G}_{n}$, with $n+1$ distinguished vertices $x_{n, 0}, x_{n, 1}, \ldots, x_{n, n}$, obtain $\mathcal{G}_{n+1}$ by decorating $\mathcal{G}_{n}$ as follows.

(a) For $i=0,1, \ldots, n$, attach to $x_{n, i}$ a path of length $\left\lceil\alpha_{i} b_{n+1}\right\rceil$ and denote the last vertex of this path by $x_{n+1, i}$

(b) to the vertex $x_{n, n}$, attach an additional path of length $\left\lceil\alpha_{n+1} b_{n+1}\right\rceil$, and denote the last vertex of this path by $x_{n+1, n+1}$,

(c) for $i=1, \ldots, n$, link $x_{n+1,0}$ and $x_{n+1, i}$ by a path of length $\left\lceil b_{n+1}^{7 / 8}\right\rceil$.

To show that if $b_{n} \rightarrow \infty$ sufficiently fast as $n \rightarrow \infty$, the graph $\mathcal{G}$ gets coexistence region $\left\{\alpha_{1}, \alpha_{2}, \ldots\right\}$, is now a completely straightforward modification of the proof of Theorem 3.1.

\section{A positive result}

In the proof of Theorem 3.2, we required that the candidate coexistence set $\left\{\alpha_{1}, \alpha_{2}, \ldots\right\}$ could be written as an increasing unbounded sequence, which is tantamount to saying that it has no accumulation points. This condition is certainly not necessary for a countable set to arise as a coexistence region for some graph, but we do not know whether it can simply be removed.

One could ask for graphs with more exotic coexistence regions, such as for instance examples whose coexistence regions are uncountable with zero Lebesgue measure. That kind of behavior is, however, ruled out by the following result. For a set $A \subseteq \mathbb{R}$, write $A^{c}$ for its complement and $\partial A$ for its boundary.

Theorem 4.1 For any graph $\mathcal{G}$, the coexistence region $\operatorname{Coex}(\mathcal{G})$ contains at most countably many points in its boundary $\partial \operatorname{Coex}(\mathcal{G})$.

Proof. For a given finite initial condition $\xi$, write $\operatorname{Coex}_{\xi}(\mathcal{G})$ for the set of $\lambda$ 's such that infinite coexistence has positive probability with initial condition $\xi$. Since $\mathcal{G}$ is countable, there are only countably many finite initial conditions $\xi$, and in order to prove the theorem it therefore suffices to show for any $\xi$ that $\operatorname{Coex}_{\xi}(\mathcal{G}) \cap \partial \operatorname{Coex}_{\xi}(\mathcal{G})$ is countable. 
Fix $\xi$. For the two-type Richardson model on $\mathcal{G}$ with parameters $\lambda_{1}=1$ and $\lambda_{2}=\lambda$, and initial condition $\xi$, let $\theta_{1}(\lambda)$ denote the probability that infection 1 reaches only finitely many sites, and let $\theta_{2}(\lambda)$ denote the probability that infection 2 reaches infinitely many. We may assume that $\mathcal{G}$ is infinite (otherwise the statement of the theorem is trivial), in which case we have $\theta_{1}(\lambda) \leq \theta_{2}(\lambda)$, with

$$
\begin{cases}\theta_{1}(\lambda)=\theta_{2}(\lambda) & \text { if } \lambda \notin \operatorname{Coex}_{\xi}(\mathcal{G}) \\ \theta_{1}(\lambda)<\theta_{2}(\lambda) & \text { if } \lambda \in \operatorname{Coex}_{\xi}(\mathcal{G})\end{cases}
$$

The standard monotone coupling of the two-type Richardson model for different $\lambda$ 's (see, e.g., [5]) shows that $\theta_{1}(\lambda)$ and $\theta_{2}(\lambda)$ are increasing functions of $\lambda$, whence they can have at most countably many discontinuities.

Now define sets $A_{-}, A_{+} \subseteq \mathbb{R}$ as

$$
A_{-}=\left\{\lambda \in \operatorname{Coex}_{\xi}(\mathcal{G}):(\lambda-\varepsilon, \lambda) \nsubseteq \operatorname{Coex}_{\xi}(\mathcal{G}) \text { for all } \varepsilon>0\right\}
$$

and

$$
A_{+}=\left\{\lambda \in \operatorname{Coex}_{\xi}(\mathcal{G}):(\lambda, \lambda+\varepsilon) \nsubseteq \operatorname{Coex}_{\xi}(\mathcal{G}) \text { for all } \varepsilon>0\right\} .
$$

For $\lambda^{*} \in A_{-}$, we see that

$$
\lim _{\lambda \nearrow \lambda^{*}} \theta_{1}(\lambda)=\lim _{\lambda \nearrow \lambda^{*}} \theta_{2}(\lambda)
$$

by considering a subsequence of $\lambda$ 's beloning to $\neg \operatorname{Coex}_{\xi}(\mathcal{G})$. Hence,

$$
\begin{aligned}
\lim _{\lambda \nearrow \lambda^{*}} \theta_{2}(\lambda) & =\lim _{\lambda \nearrow \lambda^{*}} \theta_{1}(\lambda) \\
& \leq \theta_{1}\left(\lambda^{*}\right) \\
& <\theta_{2}\left(\lambda^{*}\right)
\end{aligned}
$$

so that $\theta_{2}(\lambda)$ has a discontinuity at $\lambda=\lambda^{*}$, and it follows that $A_{-}$is countable.

We similarly get for any $\lambda^{*} \in A_{+}$that $\theta_{1}(\lambda)$ has a discontinuity at $\lambda=\lambda^{*}$, so that $A_{+}$is countable as well. Note finally that

$$
\operatorname{Coex}_{\xi}(\mathcal{G}) \cap \partial \operatorname{Coex}_{\xi}(\mathcal{G})=A_{-} \cup A_{+},
$$

which gives the desired conclusion that $\operatorname{Coex}_{\xi}(\mathcal{G}) \cap \partial \operatorname{Coex}_{\xi}(\mathcal{G})$ is countable.

\section{References}

[1] Deijfen, M. and Häggström, O. (2005) The initial configuration is irrelevant for the possibility of mutual unbounded growth in the two-type Richardson model, Comb. Probab. Computing, to appear.

[2] Deijfen, M., Häggström, O. and Bagley, J. (2004) A stochastic model for competing growth on $\mathbb{R}^{d}$, Markov Proc. Relat. Fields 10, 217-248.MR2005g:60161

[3] Garet, O. and Marchand, R. (2005) Coexistence in two-type first-passage percolation models, Ann. Appl. Probab. 15, 298-330. MR2115045 
[4] Häggström, O. and Pemantle, R. (1998) First passage percolation and a model for competing spatial growth, J. Appl. Probab. 35, 683-692.MR2000f:60153

[5] Häggström, O. and Pemantle, R. (2000) Absence of mutual unbounded growth for almost all parameter values in the two-type Richardson model, Stoch. Proc. Appl. 90, 207-222. MR2002b:60174

[6] Hoffman, C. (2005) Coexistence for Richardson type competing spatial growth models, Ann. Appl. Probab. 15, 739-747. MR2005m:60235

[7] Lundin, F. (2003) Omniparametric simulation of the two-type Richardson model, licentiate thesis 2003:6, Dept. of Mathematics, Chalmers and Göteborg University. 